\title{
Síndrome del hueso hambriento relacionado con hipertiroidismo
}

\author{
M. D. MOURE RODRÍGUEZ, M. LUQUE RAMÍREZ, G. LÓPEZ GALLARDO, \\ M. LÓPEZ IGLESIAS, A. GÓMEZ-PAN
}

Servicio de Endocrinología y Nutrición. Hospital Universitario de La Princesa. Madrid

HUNGRY BONE SYNDROME RELATED TO HYPERYTHYROIDISM

\begin{abstract}
RESUMEN
El síndrome del hueso hambriento es una entidad clínica que se caracteriza por la aparición de hipocalcemia, hipofosfatemia e hipomagnesemia secundaria a un aumento de su captación a nivel óseo. Es un proceso que se ha descrito en el contexto de enfermedades que actúan generando un disbalance entre la producción y la resorción, a favor de ésta última. La forma clásica de presentación, acontece tras la realización de una paratiroidectomia en pacientes con hiperparatiroidismo (HPT), siendo su clínica la relacionada fundamentalmente con la caída de los niveles plasmáticos de calcio. Aunque menos habitual, ha sido descrito tras tratamiento quirúrgico de entidades clínicas que cursan con un exceso de hormonas tiroideas, siendo la forma más frecuente la enfermedad de Graves-Basedow. Presentamos un caso de este síndrome tras tiroidectomía en un paciente con hipertiroidismo primario por enfermedad de Graves, realizando una breve revisión de los aspectos fisiopatológicos y manejo del cuadro.
\end{abstract}

PALABRAS CLAVE: Hipocalcemia. Tiroidectomía. Enfermedad Graves. Hipoparatiroidismo. Hipertiroidismo.

\begin{abstract}
Hungry bone syndrome is a common clinical entity which is accompanying of hypocalcemia, hypomagnesemia, and hipophosphatemia, results from an increase in bone formation. It is related to a pathological scenario which causes an imbalance between osteoclast-mediated bone resorption and osteoblast-mediates bone formation, favouring the latter. Its classic presentation occurs after parathyroidectomy in hyperparathyroydism's patients. Its clinical features are largely due to plasmatic calcium levels reduction. Hungry bone syndrome is frequent in hyperparathyroid's patients who have development bone disease before surgery. Even less frequent, it has also been described after thyroydectomy in patients with hyperthyroidism. We hereby report a case of hungry bone syndrome in one patient who suffers a Graves' disease. Then, we will provide a brief review of pathogenesis and therapeutic features.
\end{abstract}

KEY WORDS: Hypocalcemia. Thyrodectomy. Graves' disease. Hyperthyroidism. Hyperparathyroidism.

Moure Rodríguez MD, Luque-Ramírez M, López Gallardo G, López Iglesias M, Gómez-Pan A. Síndrome del hueso hambriento relacionado con hipertiroidismo. An Med Interna (Madrid) 2006; 23: 326-328.

\section{INTRODUCCIÓN}

La fisiopatología del síndrome del hueso hambriento (SHH) está relacionada con un disbalance entre la formación y resorción ósea, que se asocia a hipocalcemia, hipofosfatemia e hipomagnesemia (1). Este síndrome se puede observar tras la cirugía de pacientes con hiperparatiroidismo (HPT) primario, así como en el HPT terciario de la insuficiencia renal crónica, y en menor medida tras tratamiento de procesos que cursan con exceso de hormonas tiroideas circulantes (2). En el HPT, existe un exceso de hormona paratiroidea (PTH) que estimula la actividad osteoclástica causando desmineraliza- ción de la matriz ósea y liberando calcio al torrente sanguíneo. Tras paratiroidectomia, los niveles séricos de PTH caen dramáticamente, por ello, la resorción ósea inducida por la PTH cesa, mientras que la actividad osteoblástica continúa dando como resultado un incremento en la captación ósea de calcio, fosfato y magnesio, apareciendo así el SHH (3).

Las hormonas tiroideas actúan sobre el hueso acelerando los ciclos de remodelado, con predominio de la resorción ósea. El descenso de hormonas tiroideas tras la cirugía condiciona, una situación de disbalance óseo, favoreciendo la aparición del SHH (4). A continuación presentamos un ejemplo de esta entidad en relación con el tratamiento quirúrgico de un

Trabajo aceptado: 9 de enero de 2006 
hipertiroidismo primario y realizamos una revisión de la literatura al respecto.

\section{CASO APORTADO}

Mujer de 30 años con hipertiroidismo primario por enfermedad de Graves-Basedow de 4 años de evolución. Tras la presentación de una reacción cutánea en relación con el manejo inicial con el fármaco antitiroideo metimazol, se inició tratamiento con propiltiouracilo, y posteriormente, con litio sin alcanzar control adecuado de los niveles de hormonas tiroideas. Debido a la presencia de oftalmopatía severa se decide como tratamiento definitivo la cirugía, presentando en dicho momento los siguientes niveles de hormonas tiroideas: TSH $0,01 \mu \mathrm{U} / \mathrm{ml}(0,35-4,94 \mu \mathrm{U} / \mathrm{ml}), \mathrm{T} 4 \mathrm{~L} 2,7 \mathrm{ng} / \mathrm{dl}(0,70-1,48 \mathrm{ng} / \mathrm{dl})$.

Se practica una tiroidectomía total. Tras suspender sueroterapia, y tras 5 horas del inicio de tolerancia oral, la paciente refiere parestesias y espasmo carpo-pedal. Se solicitan niveles de calcio iónico séricos con resultado de 3,6 mg/dl. Con el diagnóstico de hipocalcemia aguda, se procede a la administración de gluconato cálcico intravenoso, con mejoría sintomática del cuadro. A las 24 horas se objetiva un calcio iónico de $4,4 \mathrm{mg} / \mathrm{dl}$, decidiéndose suspender su administración intravenosa y proceder a la suplementación de calcio y calcitriol oral.

La paciente se mantuvo asintomática 48 horas, momento en el que presenta de nuevo espasmo carpo-pedal. Se reintroduce de nuevo gluconato cálcico intravenoso tras lo cual cede la sintomatología, reinstaurándose tratamiento oral, que se acompaña de la aparición nuevamente de espasmos musculares, objetivándose un calcio iónico de $3,6 \mathrm{mg} / \mathrm{dl}$. Tras tratamiento intravenoso, la paciente se mantiene asintomática. A pesar del aporte adecuado de calcio y dosis altas de calcitriol oral, continúa manteniendo hipocalcemia a las 48 horas del comienzo del mismo (calcio iónico: $3,2 \mathrm{mg} / \mathrm{dl}$ ). Al día siguiente se solicita nueva analítica (Tabla I), en la que se objetivan niveles de calcio y magnesio disminuidos, PTH suprimida e hiperfosfatemia.

Ante estos resultados y con una presunción diagnóstica multifactorial (hipomagnesemia secundaria a síndrome de hueso hambriento por hipertiroidismo severo, hipoparatiroidismo transitorio postquirúrgico secundario a hipomagnesemia e hiperfosfatemia secundaria a sobredosificación de calcitriol), se ajusta el tratamiento iniciándose suplementación oral con magnesio y calcio, disminuyéndose el aporte de calcitriol, con lo que se consigue normalización clínica y analítica, siendo dada de alta hospitalaria en las siguientes 24 horas.

\section{DISCUSIÓN}

El síndrome del hueso hambriento, se caracteriza por unos niveles persistentemente disminuidos de calcio sérico, junto con niveles bajos o normales de fosfato y aumento de la fos- fatasa alcalina. Estos últimos son marcadores de un incremento de la actividad osteoblástica (5). La hipocalcemia aguda desencadena una hiperestimulación del sistema nervioso periférico manifestándose como parestesias, fasciculaciones y tetania, como las observadas en nuestra paciente. Este síndrome ha sido descrito en relación a múltiples procesos de los cuales el más frecuente es la paratiroidectomia (Tabla II) $(6,7)$.

La PTH aumenta la resorción ósea, estimulando la producción de citoquinas por parte de los osteoblastos que a su vez estimulan a los osteoclastos, para que desmineralicen la matriz ósea, liberando calcio hacia el torrente sanguíneo (8). En la actualidad, las manifestaciones esqueléticas del HPT son relativamente leves, siendo raras la osteítis fibrosa quística, lesiones quísticas, tumores y fracturas patológicas. Sin embargo, el aumento en el recambio óseo sigue siendo reflejado en el aumento de marcadores de formación ósea, como el aumento de la fosfatasa alcalina, y de resorción ósea, como el colágeno. Mientras que la resorción ósea es mayor que la formación ósea en el hueso cortical, se mantiene estable el volumen de hueso esponjoso y la estructura trabecular está preservada. Tras la intervención quirúrgica, el hueso sigue estando sometido a la actividad ostoblástica produciéndose una captación de calcio, fósforo y magnesio del torrente sanguíneo con la consecuente disminución de sus niveles séricos. Factores predictores del desarrollo del $\mathrm{SHH}$, son la presencia de un adenoma mayor de $5 \mathrm{~cm}$ de diámetro, niveles preoperatorios elevados de PTH, calcio y fosfatasa alcalina, la osteítis fibrosa quística y una edad mayor de 60 años $(9,10)$. Con el incremento de la detección del HPT en estadio asintomático, la incidencia del SHH está disminuyendo. Por ello, hoy en día, se ve asociado con mayor frecuencia a paratiroidectomia por $\mathrm{HPT}$ terciario. Otro proceso relacionado con el $\mathrm{SHH}$, es la hiperproducción de hormonas tiroideas.

La enfermedad de Graves, es la causa más común de tirotoxicosis, con una prevalencia que oscila entre el $60-80 \%$ de los casos de hipertiroidismo primario. Para poder comprender la etiopatogenia del $\mathrm{SHH}$ en esta situación es imprescindible conocer los efectos de dichas hormonas a nivel óseo (11). Las hormonas tiroideas son necesarias para el reclutamiento, maduración y la actividad de osteoblastos y osteoclastos. Modulan el proceso de remodelado e inducen cambios secundarios en las concentraciones de calcio, PTH y vitamina D. Su exceso ocasiona un desequilibrio en el tejido óseo, caracterizado por la aceleración de los ciclos de remodelado con predominio de la resorción. El incremento de la actividad osteoclástica provoca

TABLA I

EVOLUCIÓN ANALÍTICA DEL PACIENTE

\begin{tabular}{|c|c|c|c|c|c|c|c|}
\hline Día & $\begin{array}{c}\text { Ca iónico } \\
\text { mg/dl }\end{array}$ & $\begin{array}{l}\text { Ca total } \\
m g / d l\end{array}$ & $\begin{array}{c}P \\
m g / d l\end{array}$ & $\begin{array}{c}\mathrm{Mg} \\
\mathrm{mg} / \mathrm{dl}\end{array}$ & $\begin{array}{c}\text { Proteínas totales } \\
\mathrm{g} / \mathrm{dl}\end{array}$ & $\begin{array}{l}\text { Albúmina } \\
\text { g/dl }\end{array}$ & $\begin{array}{c}\text { PTH } \\
\mathrm{pg} / \mathrm{ml}\end{array}$ \\
\hline 1 & 3,6 & & & & & & \\
\hline 2 & 4,4 & & & & & & \\
\hline 3 & 3,6 & & & & & & \\
\hline 5 & 3,2 & & & & & & \\
\hline 6 & & 7,1 & 7,2 & 1,48 & & & 3,63 \\
\hline $\begin{array}{l}7 \\
8\end{array}$ & 3,6 & 8,4 & 5,8 & 1,74 & 7,7 & 3,7 & \\
\hline
\end{tabular}


TABLA II

ETIOLOGÍA DEL SÍNDROME DEL HUESO HAMBRIENTO

\section{Paratiroidectomia}

2. Tiroidectomia en pacientes con hipertiroidismo

3. Corrección de acidosis metabólica

4. Metástasis osteoblásticas del cáncer de próstata

TABLA III

CAUSAS DE HIPOCALCEMIA TRAS PARATIROIDECTOMÍA

1. Hipoparatiroidismo transitorio

2. Hipoparatiroidismo permanente

3. Síndrome del hueso hambriento

\section{Hipomagnesemia}

\section{Liberación de calcitonina tras cirugía}

elevación del calcio sérico que inhibe la secreción de PTH, lo que a su vez disminuye la formación de $1,25-(\mathrm{OH})_{2}$ vitamina $\mathrm{D}$. La hipercalciuria secundaria a la disminución de PTH y de la absorción intestinal de calcio, por el descenso de vitamina D, condicionan un resultado final del balance cálcico negativo. La determinación de los marcadores bioquímicos de remodelado óseo pone de manifiesto un estado de alto turnover que se mitiga una vez alcanzado el eutiroidismo.

La cantidad de hueso perdida en la evolución de un hipertiroidismo oscila del 5 al $17 \%$ según las series. Esta pérdida ósea es una combinación de mecanismos reversibles e irreversibles. El componente irreversible incluye la pérdida de ele- mentos estructurales, debido a perforaciones, adelgazamiento trabecular y disminución de la cortical por resorción endocortical. La parte reversible se debe a una expansión del espacio de remodelado por el aumento en el número de unidades de remodelado activas. A pesar de la potencial reversibilidad de la pérdida de masa ósea, diversos estudios epidemiológicos señalan que los pacientes hipertiroideos presentan un riesgo aumentado de desarrollar osteoporosis y fracturas por fragilidad. Cuanto mayor es el periodo de tiempo en hipertiroidismo mayores serán las probabilidades de desarrollo del SHH. La incidencia de hipocalcemia postquirúrgica se situa entorno al $12 \%$. La incidencia de hipocalcemia tras tiroidectomía varía según las series entre el 0,6 y el 40\% (12,13). Estas diferencias son debidas en gran parte a la manera de diagnosticar la entidad, bien por métodos clínicos, o mediante la determinación de calcio iónico, calcio total o PTH (14). Analíticamente, en el SHH encontramos hipocalcemia persistente, fosfatemia normal o baja, niveles séricos de fosfatasa alcalina aumentados y calciuria disminuida. El tratamiento del SHH consiste en la administración de gluconato de calcio iv, bajo vigilancia electrocardiográfica. Debemos monitorizar el calcio sérico frecuentemente, con el fin de observar la respuesta al tratamiento y decidir el momento de la utilización de la vía oral $(15,16)$.

Los pacientes con arritmias cardíacas o en tratamiento con digoxina requieren monitorización cardiaca continua. El tratamiento con calcio y vitamina D por 1-2 días previos a la paratiroidectomía, ayuda a prevenir el desarrollo de hipocalcemia. En pacientes en postoperatorio inmediato de paratiroidectomía, o SHH, se debe administrar calcio y vitamina $\mathrm{D}$ por vía oral de manera temprana (17).

Si la sintomatología es refractaria o se detecta hipomagnesemia, se administrará magnesio oral o parenteral según la gravedad del paciente. En sujetos asintomáticos, se recomienda la suplementación diaria de 1-4 g de calcio elemental fraccionado en varias dosis.

\section{Bibliografía}

1. Bhattachary A, Bukler HM, New JP. Hungry bone syndrome-Revised. J R Coll Physicians Edinb 2002; 32: 83-6.

2. Campusano C, López JM. Complete recovery of hungry bone syndrome using intravenous calcium infusion. Report of one case. Rev Med Chil 2003; 131: 779-84

3. Krempl GA, Medina JE, Bouknight AL. Surgical management of parathyroids. Otolaryngol Clin North Am 2003; 36: 217-28.

4. Campusano C. Enfrentamiento clínico del paciente con osteoporosis. Boletín de la escuela de medicina [revista electrónica] 1999; 28 (1-2) [consultado 24-11-2005]: Disponible en: http://escuela.med.puc.cl/publ/Boletin/ Osteoporosis/IndiceOsteoporosis.html

5. Gundberg CM. Biochemical markers of bone formation. Clin Lab Med 2000; 20: 489-501.

6. Murray TM, Rao LG, Divieti P, Bringhurst FR. Parathyroid hormone secretion and action: evidence for discrete receptors for the carboxylterminal region and related biological actions of carboxyl- terminal ligands. Endocr Rev 2005; 26: 78-113.

7. Ehrlich PJ, Lanyon LE. Mechanical strain and bone cell function: a review. Osteoporosis Int 2002; 13: 688-700.

8. Manzano F, Krouham A. Hiperparatiroidismo primario. Cir Ciruj 2002; 7:150-6.

9. Sarko J. Bone and mineral metabolism. Emerg Med Clin North Am

2005; 23: 703-21

10. Martínez G, Jodar E, Azriel S, Hawkins F. Alteraciones metabólicas óseas de las enfermedades endocrinológicas. Medicine 2002; 84: 451624.

11. Mittendorf EA, Merlino JI, McHenry CR. Post-parathyroidectomy hypocalcemia: incidence, risk factors, and management. Am Surg 2004; 70:114-9.

12. Herranz-González J, Gavilán J, Martínez-Vidal J, Gavilan G. Complications following thyroid surgery. Arch Otolaryngol Head Neck Surg 1991; 117: 516-8.

13. Clark OH. Total thyroidectomy. Ann Surg 1982; 196: 361-7.

14. Karlsson MK, Gerdhem P, Aalborg HG. The prevention of osteoporotic fractures. J Bone Joint Surg Br 2005; 87: 1320-27.

15. Bringhurst FR, Demay MB, Kronenberg HM. Hormones and disorders of mineral metabolism. En: Larsen PR, Kronenberg HM, Melmed S, Polonsky KS, editors. Williams Texbookof Endocrinology. Philadelphia: Saunders, 2003: p. 1341-5.

16. Goltzman D, Cole DE. Hypoparathyroidism. En: Favus MJ, editors. Primer on the metabolic bone diseases and disorders of mineral metabolism. Philadelphia: Lippincott, Williams, 1999: p. 226-30.

17. Ariyan CE, Sosa JA. Assessment and management of patients with abnormal calcium. Crit Care Med 2004; 32 (4 Supl.): S146-54 